\title{
A Survey on Various Single Image Super Resolution Techniques
}

\author{
A.Hazarathaiah \\ Professor, Department of ECE, S V College of Engineering, Tirupati, A.P, India
}

\begin{abstract}
Super-resolution from a single frame is play an important role in many computer vision systems. Superresolution is the process of recovering a high-resolution (HR) image from single image or multiple low-resolution (LR) images of the same scene. The main aim of super resolution (SR) is to increase better visual quality of available low resolution image. The SR image approaches reconstruct a single higher-resolution image from a set of given lower-resolution images. There is a basic need for digital images of higher resolutions and quality. In this paper, we presented different existing super resolution methods, positive and negative aspects of those methods, relevant work and methods of super resolution reconstruction method. Super resolution methods which is generate high-resolution (HR) image from one or more low resolution images and various image quality metrics reviewed as measure the original image and reconstructed image.
\end{abstract}

KEYWORDS: Super Resolution, Image Reconstruction, Single Image Resolution Techniques, Resolution Enhancement

\section{INTRODUCTION}

Image super resolution is a image processing algorithms that produce high quality, high resolution (HR) images from a set of low quality, low resolution (LR) images or from a single image. The SR image reconstruction is useful in many practical cases where multiple frames of the same scene can be obtained, including medical imaging, satellite imaging, and video applications[17].The basic premise for increasing the spatial resolution in SR techniques is the availability of multiple LR images captured from the same scene. The set of source low resolution (LR) images captures only a finite amount of information from a scene; the goal of SR is to extract the independent information from each image in that set and combine the information into a single high resolution (HR) image. The requirement is of SR is that each LR image must contain some information that is unique to that image[3]. The super resolution method is to take more samples of the scene so as to get some extra information which can be used, while merging the samples to get a high resolution image. These samples can be acquired by sub-pixel shifts, by changing scene, by changing the amount of blur [14] .HR means that pixel density within an image is high, and therefore an HR image can offer more details that are important in many applications, The major advantage of the super resolution approach is that it may cost less and the existing LR imaging systems can be still utilized. Synthetic zooming of region of interest (ROI) is another important application in surveillance, forensic, scientific, medical, and satellite imaging[13]. This application is most suitable for magnifying objects in the scene such as the face of a criminal or the license plate of a car [16].

\section{CLASSIFICATION OF SUPER RESOLUTION APPROACH}

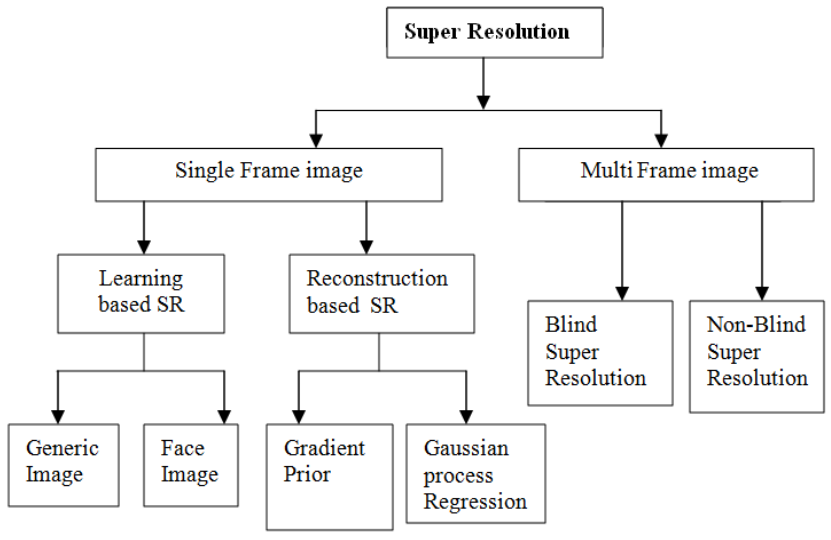

Fig-1 Classification of Super Resolution 


\section{International Journal of Innovative Research in Science, Engineering and Technology}

Vol. 1, Issue 2, December 2012

\section{RELAVANCE SURVEY ON SR TECHNIQUES FOR SINGLE IMAGE}

Feilong Cao, Miaomiao Cai, and Yuanpeng Tan [1] proposed a method that can transformed a color image from RGB color space to $\mathrm{YCbCr}$ color space. the color channels $(\mathrm{Cb}$ and $\mathrm{Cr}$ ), the bicubic interpolation method is used to upsample them. The proposed method aims to define the local linear relationship among neighboring pixels. By considering the low-rank property of the augmented matrix, the SR problem has been reformulated as the recovery of a low-rank matrix from missing and corrupted observations, which can be solved efficiently using the ALM method. The advantage of the proposed method is its ability to handle noisy data and random perturbations and also provides the feasibility and effectiveness for the real images with noisy data.

This paper [2] presents a new recovery approach for single image super resolution based on sparse. The recovered patch is a linear combination of the some patches, the noise of every patch, aggregated in the recovered patch. Sparse representation of patches can used for filter the noise in the solution. This method calculate the sparse representation of every patches and set it to the recovered high-resolution image. The dictionary that contains the details of patches, so it cant to be small. The computation using huge dictionary is take more time . In this approach the image is reconstructed from LR image to HR image using sparse representation for reduced the noise from LR image.

In paper [3] propose a algorithm for fast single image super resolution based on self-example learning and sparse representation. Basically 4 steps are done in this reconstruction of image : In first step LR image patches are converted in HR patches using sparse. We only use LR input image itself to learn dictionary without an HR images. In second step do self-example sampling for reduce the time. In third step self-example patch based dictionary learning is proposed based on KSVD (K-singular value decomposition) algorithm is allow to learn this dictionary for the sparse representation of LR input image. In last phase apply the fast single image SR algorithm for get the HR output image. This approach generate high resolution images that have similar quality to other methods and highly competitive in terms of the reconstruction performance.

In this [4] presented method of producing HR image from single LR image. In this method use a dictionary-based regression model using local self-similar example patches within the image. A first order approximation of nonlinear mapping function,learned using local self-similar example patches is applied to low resolution image pathes for obtain the high resolution image patches. This approach is more accurate and produce result with sharp details with noise.

A novel approach introduced by Priyanka Chaudhary and Bhupendra Fataniya [5] is first phase, improve a set of low resolution images using learning dictionary method and in second phase combine by projecting images onto convex sets so enhancing image by information procured from multiple images. In short in first stage enhanced multiple images via single stage SR algorithm and in second stage combined them to produce a single high resolution SR image.

This [6] paper propose a new method for image super- resolution using a local sparse model on image patches based on a new dictionary training formulation. The high-resolution deficiency image is reconstructed by the proposed super- resolution method and compensated to better preserve the high-frequency details of images. The given results of this method show visual, PSNR and SSIM improvements. This method performs visually much better than bicubic interpolation, and on some images considerably better than Yang's method and produces sharper results and preserves more image details.

A fast algorithm proposed in [7] using mapping functions to generate SR images. By splitting the feature space into numerous subspaces and collecting sufficient training exemplars to learn simple regression functions, the proposed approach generates high quality SR images with sharp edges and rich textures and gives effective and efficient algorithm for reconstruct the images.

In paper [8] solved the problem of generating a super- resolved version of a LR textual image using sparse representation that suggest image patches from suitable dictionary. This paper proposed a multiple learned dictionaries based clustered sparse coding approach for single text image super resolution. A large HR/LR patch pair database is collected from a set of high quality character images and partitioned into clusters for perform clustering algorithm. This approach provides image that minimizes the reconstruct error and applied to text images. The propose method have better visual quality compare of others method like bicubic interpolation that generates blur effects. They produce clear and sharper character.

In [9] for a single frame super resolution method using CGP (Cartesian Genetic Programming).This method is to learn relationship of pixel values between high-resolution image and low resolution image using CGP.A single pixel and its neighbor pixels of the LR input image are set to the inputs of CGP and then pixel values of SR image are obtained from calculated outputs of CGP. Multiple CGP can improve the quality of SR image and also suitable to parallel processing for reducing computational cost.

In paper [10] propose a novel single-image super resolution algorithm by integrating the exemplar-based technique with the reconstruction-based formulation .They exploit the idea of the exemplar-based approach by searching similar patches and forming them into a group and used for solving the image denoising problem. Then they place these groups into the classical reconstruction-based formulation for estimating the fine resolution image. The experiments 


\section{International Journal of Innovative Research in Science, Engineering and Technology}

Vol. 1, Issue 2, December 2012

demonstrate the high quality super resolution results by using the proposed algorithm, which does not require any external dataset.

Jianhong Li and Xiaocui Peng [11] Proposed a method based on the traditional methods that learn through training sets, this method increases the priority of low-frequency images' gradient region and learns from gradient region. They extract features from every gradient image fragment, and then find the closest matching fragments to determine the high-frequency of target image. At first, we get horizontal and vertical gradient images of the input image, then combine these two images together to get the final high- resolution image. Using this approach, the target image has sharper edges and higher quality.

Single Image super resolution [12] using improved example- based approach for reduce the time for real time application. Classify method is used to mark the high-frequency patches of the low resolution image with labels. During the SR, distance between each matching patches of LR image and middle- frequency patches of training set are computed and candidate patch is set with minimum distance. Few candidates patches are selected ,while for flat patches matching step can be canceled, directly replacing high-resolution patches by enlarge of LR patches.

Appendix A summarizes above approaches providing addition knowledge about overview and limitations of each approach.

\section{CONCLUSION AND FUTURE WORK}

This paper presents some Super resolution algorithms and techniques used in single imaging. Single imaging algorithms are mostly application based but the fact is all of these algorithms are not applicable for all applications. It also faces some difficulties in optimal camera sensor designing, high dynamic range image super resolution etc .

In this survey paper, our goal is to offer new perspectives and out looks of SR imaging research, besides giving an updated overview of existing SR algorithms. It can overcome or compensate the inherent hardware limitations of the imaging system to provide amore clear image with a richer and informative content. We have provided a survey of the current state of super-resolution research, covering the past, present, and future of the problem. There must be considerations like if more than one input images are present then use multi frame super resolution approach and if one or more high resolution training images are available then use single image super resolution approach.

In future develop SR enhancement of random scenes containing global, multiple independent and individual motion, occlusions, transparency etc. is a main focus of SR research. Other issues in the SR techniques to improve their performance are currently focused on the color SR algorithm and the application to compression systems. It is necessary to extend the current SR algorithm to a real-world color imaging system. We covered recent analyses on the limits of super resolution algorithms and finally discussed potential future directions for research.

APPENDIX-A

TABLE I : A Related Survey On Single Image Super Resolution

\begin{tabular}{|c|c|c|c|c|}
\hline Year & Title & Method & Overview & Limitations \\
\hline \begin{tabular}{|l} 
IEEE/ \\
2015
\end{tabular} & $\begin{array}{l}\text { Image Interpolation } \\
\text { via Low-Rank } \\
\text { Matrix Completion } \\
\text { and Recovery [1] }\end{array}$ & $\begin{array}{l}\text { Interpolation } \\
\text { using } \\
\text { low-rank matrix }\end{array}$ & $\begin{array}{l}\text { - To handle noisy data and random } \\
\text { perturbations } \\
\text { robustly } \\
\text { - To estimate the missing pixels and to } \\
\text { explore }\end{array}$ & $\begin{array}{l}\text { - Generate serrated and } \\
\text { blurred edges as recovery } \\
\text { effects }\end{array}$ \\
\hline $\begin{array}{l}\text { IEEE/ } \\
2015\end{array}$ & $\begin{array}{l}\text { Integrated Single } \\
\text { Image Super } \\
\text { Resolution Based } \\
\text { on Sparse } \\
\text { Representation [2] }\end{array}$ & $\begin{array}{l}\text { Integrated } \\
\text { method } \\
\text { using dictionary } \\
\text { of patches and } \\
\text { sparse } \\
\text { representation }\end{array}$ & $\begin{array}{l}\text { - Uses linear combination of low - high } \\
\text { dictionary } \\
\text { patches } \\
\text { - Noise of every patch recovered the patch } \\
\text { by using sparse } \\
\text { - Desired computational complexity }\end{array}$ & $\begin{array}{l}\text { - Complexity is very } \\
\text { high } \\
\text { - Time consuming }\end{array}$ \\
\hline $\begin{array}{l}\text { IEEE/ } \\
2014\end{array}$ & $\begin{array}{l}\text { Fast Single Image } \\
\text { Super-Resolution } \\
\text { via Self-Example } \\
\text { Learning and } \\
\text { Sparse }\end{array}$ & $\begin{array}{l}\text { Self example } \\
\text { learning and } \\
\text { sparse } \\
\text { representation }\end{array}$ & $\begin{array}{l}\text { - Patches for dictionary learning are } \\
\text { efficiently } \\
\text { sampled } \\
\text { - Without an external a large collection of } \\
\text { high resolution }\end{array}$ & $\begin{array}{l}\text { - Computational } \\
\text { efficiency is decrease }\end{array}$ \\
\hline
\end{tabular}




\section{International Journal of Innovative Research in Science, Engineering and Technology}

Vol. 1, Issue 2, December 2012

\begin{tabular}{|c|c|c|c|c|}
\hline \begin{tabular}{|l} 
IEEE/ \\
2014
\end{tabular} & $\begin{array}{l}\text { Single Image } \\
\text { Super-Resolution } \\
\text { Using } \\
\text { Dictionary-Based } \\
\text { Local Regression } \\
{[4]}\end{array}$ & $\begin{array}{l}\text { Dictionary based } \\
\text { regression model } \\
\text { using self- } \\
\text { similarity patch }\end{array}$ & $\begin{array}{l}\text { - Improved accuracy and recover local } \\
\text { texture } \\
\text { details ,sharp edge } \\
\text { - Running on that contain diverse textures, } \\
\text { noise or other artifacts }\end{array}$ & $\begin{array}{l}\text { - Produce more natural } \\
\text { results with sharp details } \\
\text { with noise }\end{array}$ \\
\hline $\begin{array}{l}\text { IEEE/ } \\
2014\end{array}$ & $\begin{array}{l}\text { A Robust Two- } \\
\text { Stage } \\
\text { Super-Resolution } \\
\text { Algorithm [5] }\end{array}$ & $\begin{array}{l}\text { Learning } \\
\text { dictionary } \\
\text { method using } \\
\text { projection onto } \\
\text { convex set }\end{array}$ & $\begin{array}{l}\text { - Enhanced multiple images via single stage } \\
\text { SR } \\
\text { algorithm. } \\
\text { - Combined them to produce a single high } \\
\text { resolution super resolution image. }\end{array}$ & $\begin{array}{l}\text { - Requires } \\
\text { missing image data } \\
\text { be calculated }\end{array}$ \\
\hline \begin{tabular}{|l} 
IEEE/ \\
2014
\end{tabular} & $\begin{array}{l}\text { Dictionary } \\
\text { Learning } \\
\text { for Image Super- } \\
\text { resolution [6] }\end{array}$ & $\begin{array}{l}\text { Dictionary } \\
\text { learning using } \\
\text { local sparse } \\
\text { model }\end{array}$ & $\begin{array}{l}\text { - HR image is reconstructed using proposed } \\
\text { SR } \\
\text { method } \\
\text { To better preserve the high-frequency } \\
\text { details of images }\end{array}$ & $\begin{array}{l}\text { - Exploit the property of } \\
\text { non-local self-similarities } \\
\text { in images } \\
\text { - A sparse model based } \\
\text { on } \\
\text { structural similarity use }\end{array}$ \\
\hline \begin{tabular}{|l} 
IEEE/ \\
2013
\end{tabular} & $\begin{array}{l}\text { Fast Direct Super- } \\
\text { Resolution by } \\
\text { Simple } \\
\text { Functions [7] }\end{array}$ & $\begin{array}{l}\text { Patch based } \\
\text { dictionary } \\
\text { Exemplar patche }\end{array}$ & $\begin{array}{l}\text { - Splitting the feature space into numerous } \\
\text { subspaces. } \\
\text { - Collecting sufficient training exemplars to } \\
\text { learn simple regression functions. } \\
\text { - Generates high-quality SR images with } \\
\text { sharp edges and rich textures. }\end{array}$ & $\begin{array}{l}\text { Less effective to } \\
\text { reconstruct high- } \\
\text { frequency details. }\end{array}$ \\
\hline \begin{tabular}{|l} 
IEEE/ \\
2013
\end{tabular} & $\begin{array}{l}\text { Multiple Learned } \\
\text { Dictionaries based } \\
\text { Clustered } \\
\text { Sparse Coding for } \\
\text { the } \\
\text { Super-Resolution } \\
\text { of Single Text }\end{array}$ & $\begin{array}{l}\text { Multiple learned } \\
\text { dictionaries } \\
\text { based on } \\
\text { clustered sparse } \\
\text { coding }\end{array}$ & $\begin{array}{l}\text { - HR/LR patch pair database is collected. } \\
\text { - Partition into several clusters and using } \\
\text { sparse code local patch is same as dictionary } \\
\text { generating } \\
\text { multiple sparse of LR image. } \\
\text { - Good image quality } \\
\text { - Character recognition accuracy. }\end{array}$ & $\begin{array}{l}\text { - To improve text images } \\
\text { quality in video } \\
\text { applications. }\end{array}$ \\
\hline \begin{tabular}{|l} 
IEEE/ \\
2013
\end{tabular} & $\begin{array}{l}\text { Automatic } \\
\text { Construction of } \\
\text { Single Frame } \\
\text { Super- Resolution } \\
\text { Using Cartesian } \\
\text { Genetic } \\
\text { Programming [9] }\end{array}$ & $\begin{array}{l}\text { CGP(Cartesian } \\
\text { Generic } \\
\text { Programming) }\end{array}$ & $\begin{array}{l}\text { - A single and its neighbor pixels of LR } \\
\text { input image } \\
\text { are set to the inputs of CGP. } \\
\text { - Pixel values of the SR image calculated } \\
\text { outputs of } \\
\text { CGP. } \\
\text { - Accuracy improved using multiple CGP. }\end{array}$ & $\begin{array}{l}\text { - Faster but quality is } \\
\text { less. } \\
\text { - Not suitable for multi- } \\
\text { frame because takes } \\
\text { more calculation cost }\end{array}$ \\
\hline
\end{tabular}

\begin{tabular}{|c|c|c|c|c|}
\hline \begin{tabular}{|l} 
IEEE/ \\
2013
\end{tabular} & $\begin{array}{|lr|}\text { Single } & \text { Image } \\
\text { Super- } & \\
\text { Resolution } & \text { Based } \\
\text { on Local r Self- } \\
\text { Similarity [10] }\end{array}$ & $\begin{array}{l}\text { Local Self - } \\
\text { similarity }\end{array}$ & $\begin{array}{l}\text { - Estimate the corresponding high-resolution } \\
\text { image } \\
\text { without any additional information } \\
\text { or dataset } \\
\text { 1) Grouping local similar patches } \\
\text { 2) The inferring high-resolution image based } \\
\text { on an image capturing model. }\end{array}$ & $\begin{array}{l}\text { - Patches that contains } \\
\text { scaling or rotation will } \\
\text { give better result for } \\
\text { more information from } \\
\text { LR image }\end{array}$ \\
\hline $\begin{array}{l}\text { IEEE/ } \\
2012\end{array}$ & \begin{tabular}{|l|} 
Single-Frame \\
Image \\
Super-Resolution \\
through Gradient \\
Learning [11]
\end{tabular} & $\begin{array}{l}\text { Gradient } \\
\text { Learning }\end{array}$ & $\begin{array}{l}\text { - Increases the priority of low-frequency } \\
\text { images } \\
\text { gradient region } \\
\text { - Extract features from every gradient image } \\
\text { fragment, then find closest matching } \\
\text { fragments to determine the high-freauencv }\end{array}$ & $\begin{array}{l}\text {-Horizontal and vertical } \\
\text { gradient map makes } \\
\text { learning method more } \\
\text { sensitive }\end{array}$ \\
\hline
\end{tabular}


International Journal of Innovative Research in Science, Engineering and Technology Vol. 1, Issue 2, December 2012

\begin{tabular}{|c|c|c|c|c|}
\hline $\begin{array}{l}\text { IEEE/ } \\
2010\end{array}$ & $\begin{array}{l}\text { Improved Example- } \\
\text { based Single-image } \\
\text { Super-resolution } \\
{[12]}\end{array}$ & $\begin{array}{l}\text { Example based } \\
\text { method using } \\
\text { high frequency } \\
\text { patches }\end{array}$ & $\begin{array}{l}\text { - Minimum distance between each match } \\
\text { patches } \\
\text { and middle frequency patches are computed } \\
\text { - Patches labeled with non-edge and flat } \\
\text { patches are canceled } \\
\text { - Directely replacing high resolution patches. } \\
\text { - Requires much less processing time. }\end{array}$ & $\begin{array}{l}\text { - Difficult to implement } \\
\text { in } \\
\text { real-time SR applica- } \\
\text { tions and find obtained } \\
\text { clustering result is more } \\
\text { perfect. }\end{array}$ \\
\hline
\end{tabular}

\section{REFERENCES}

[1] Feilong Cao, Miaomiao Cai, and Yuanpeng Tan,” Image Interpolati on via Low-Rank Matrix Completion and Recovery”,IEEE 2015

[2] Mehdi Khademloo and Mansoor Rezghi ”Integrated Single Image Super Resolution Based on Sparse Representation”,IEEE,2015.

[3] Zhiliang Zhu, Fangda Guo and Hai Yu, and Chen Chen"Fast Single Image Super-Resolution via Self-Example Learning and Sparse Representation”,IEEE,2014.

[4] Sundaresh Ram and Jeffrey J. Rodriguez“Single Image SuperResolution Using Dictionary-Based Local Regression”,IEEE,2014.

[5] Priyank Chaudhary, and Bhupendra Fataniya“"A Robust Two-Stage Super Resolution Algorithm” ,IEEE,2013.

[6] LI Juan, WUJin, YANG Shen and LIU Jin ,” Dictionary Learning for Image Super-resolution”,IEEE,2013.

[7] Chih-Yuan Yang and Ming-Hsuan Yang "Fast Direct Super-Resolution by Simple Functions”,IEEE.2013.

[8] Rim Walha, Fadoua Drira, Franck Lebourgeois, Christophe Garcia and Adel M. Alimi "Multiple Learned Dictionaries based Clustered Sparse Coding for the Super-Resolution of Single Text Image",

IEEE,2013. 